\title{
Finite Difference and Ratio Operations: Representation of Numerical Data on a Pair of Variables by a Polynomial Curve
}

\author{
Dhritikesh Chakrabarty \\ Department of Statistics, Handique Girls' College, Guwahati, Assam, India \\ dhritikesh.c@rediffmail.com,dhritikeshchakrabarty@gmail.com
}

\begin{abstract}
The recently introduced approach to interpolation which consists of the representation of numerical data by a suitable polynomial curve and then to compute the value of the dependent variable from the curve corresponding to any given value of the independent variable leads to the necessity of a method/formula for representing a given set of numerical data on a pair of variables by a suitable polynomial curve. One method, in addition to the existing methods, has been developed for representing a set of numerical data on a pair of variables by a suitable polynomial curve. The method developed here, which is simpler than the earlier ones, is based on two numerical operations namely finite difference operation and ratio operation. This paper describes the development of the method with numerical example in order to show the application of the method to numerical data.
\end{abstract}

Key words: Finite difference operation, ratio operation, polynomial curve, representation of numerical data

\section{Introduction:}

Interpolation which is a technique of determining, on the basis of a set of pairs of values of independent and dependent variables, the value of the dependent variable corresponding to a value of the independent variable lying between its two extreme of the available values [Bathe \& Wilson 1976, Hummel 1947, Wisniewski et al., 1930] is basically based on the representation of the given numerical data by a polynomial curve of suitable degree. A number of interpolation formulas are available in the literature of numerical analysis [Traub 1964, Bathe \& Wilson 1976, Wisniewski 1930, Hummel et al., 1947] 
The existing formulas for numerical interpolation [Hummel 1947, Erdos \& Turan 1938, Bathe \& Wilson 1976, Whittaker \& Robinson 1967, Bathe \& Wilson 1976, Quadling 1966, Mills 1977 , Echols 1893, Revers \& Michael et al., 2000] suffer from one demerit in their computational parts. In computing the values of the dependent variable to be interpolated corresponding to a number of values of the independent variable, numerical data are to be put in the formula afresh for each interpolation i.e. if it is wanted to interpolate the values of the dependent variable corresponding to a number of values of the independent variable by a suitable existing interpolation formula then it is required to put the numerical data in the formula afresh in computing the value of the dependent variable corresponding to each value of the independent variable and hence the volume of works in numerical computations of the values of the dependent variable based on the given data becomes larger and larger as the number of values to be interpolated becomes larger. In order to get rid of the numerical computations based on the afresh application/substitution of numerical data in the formula, an approach was introduced which consists of the representation of the given numerical data by a suitable polynomial curve and then to compute the value of the dependent variable from the polynomial curve corresponding to any given value of the independent variable [Chakrabarty 2016a]. In this approach, a number of formulas [Chakrabarty $2016 a$, 2016c, 2016 - 17, 2017a; Das \& Chakrabarty 2016a, 2016b, 2016c, 2016d] and some methods [Chakrabarty 2016b, 2016d, 2017b; Das \& Chakrabarty 2016e, 2016f, 2017a, 2017b] have already been derived/developed for representing a set of the numerical data on a pair of variables by a polynomial curve. Moreover, some methods have already been developed for representing a set of the numerical data on a pair of variables by some non-polynomial curves [Das \& Chakrabarty 2017c, 2017d, 2018a, 2018b; Das, Chakrabarty \& Kashyap 2017, 2018]. One method, in addition to the existing methods, has been developed for representing a set of numerical data on a pair of variables by a suitable polynomial curve. The method developed here, which is simpler than the earlier ones, is based on two numerical operations namely finite difference operation and ratio operation. This paper describes the development of the method with numerical example in order to show the application of the method to numerical data.

\section{Representation of Numerical Data by Polynomial Curve:}

Let

$$
\left(x_{0}, y_{0}\right),\left(x_{1}, y_{1}\right),\left(x_{2}, y_{2}\right), \ldots \ldots \ldots \ldots,\left(x_{n}, y_{n}\right)
$$


be n points in $X-Y$ plane

The objective is to represent these $\mathrm{n}$ points by a polynomial of the form

$$
y=f(x)=a_{0}+a_{1} x+a_{2} x^{2}+\ldots \ldots \ldots \ldots \ldots+a_{n} x^{n}, a_{n} \neq 0
$$

where $a_{0}, a_{1}, a_{2}, \ldots \ldots \ldots \ldots \ldots, a_{n}$ are parameters.

In this study, two operators namely difference operator (denoted by $\Delta$ ) and radio operator (denoted by $R[$.

: .] have been used in the derivation of the method aimed at.

The operator $\Delta$ has been defined with respect to the suffix variable as

$$
\Delta x_{i}=x_{i+1}-x_{i} \quad \& \quad \Delta y_{i}=y_{i+1}-y_{i}
$$

while the operator $R[$. : . .] has been defined as

$$
R[f(.): g(.)]=\frac{f(.)}{g(.)}
$$

i.e. $R[f():. g()$.$] denotes the ratio of f() \& g.($.$) ;$

Case-1 (Representation of two points by a linear curve):

Let

$$
\left(x_{0}, y_{0}\right),\left(x_{1}, y_{1}\right)
$$

be two points in $X-Y$ plane.

It is possible to draw one straight line such that the line passes through the two points.

Let the straight line (i.e. linear curve) passing through the two points be

$$
y=a+b x
$$

where $a \& b$ are the parameters to be determined the two points.

Since the line passes through the two points

therefore,

$$
\begin{aligned}
y_{0} & =a+b x_{0} \\
\& \quad y_{1} & =a+b x_{1}
\end{aligned}
$$

This yield,

$$
\Delta y_{0}=b \Delta x_{0} \quad \text { i.e. } \quad b=\frac{\Delta y_{0}}{\Delta x_{0}}
$$

Therefore,

$$
a=y_{0}-b x_{0}=y_{1}-b x_{1}
$$




$$
\text { i.e. } \quad b=\frac{\Delta y_{0}}{\Delta x_{0}} \quad a=y_{0}-\left(\frac{\Delta y_{0}}{\Delta x_{0}}\right) x_{0}=y_{1}-\left(\frac{\Delta y_{0}}{\Delta x_{0}}\right) x_{1}
$$

Hence the straight line passing through the two points $\left(x_{0}, y_{0}\right) \&\left(x_{1}, y_{1}\right)$ is

$$
y=\left\{y_{0}-\left(\frac{\Delta y_{0}}{\Delta x_{0}}\right) x_{0}\right\}+\left(\frac{\Delta y_{0}}{\Delta x_{0}}\right) x=\left\{y_{1}-\left(\frac{\Delta y_{0}}{\Delta x_{0}}\right) x_{1}\right\}+\left(\frac{\Delta y_{0}}{\Delta x_{0}}\right) x
$$

Case-2 (Representation of three points by a quadratic curve):

Let

$$
\left(x_{0}, y_{0}\right),\left(x_{1}, y_{1}\right),\left(x_{2}, y_{2}\right)
$$

be three points in $X-Y$ plane.

Let the 2nd degree curve passing through the three points be

$$
y=a+b x+c x^{2}
$$

where $a, b \& c$ are the parameters to be determined the three points.

Since the curve passes through the three points,

therefore,

$$
\begin{aligned}
& y_{0}=a+b x_{0}+c x_{0}{ }^{2}, \\
& y_{1}=a+b x_{1}+c x_{1}{ }^{2} \\
& \& y_{2}=a+b x_{2}+c x_{2}{ }^{2}
\end{aligned}
$$

This yields

$$
\begin{gathered}
\Delta y_{0}=b \Delta x_{0}+c \Delta x_{0}{ }^{2} \\
\& \Delta y_{1}=b \Delta x_{1}+c \Delta x_{1}{ }^{2}
\end{gathered}
$$

This can be expressed as

$$
\begin{aligned}
& y_{0}(1)=b+c x_{0}{ }^{2}(1) \\
& \& y_{1}(1)=b+c x_{1}^{2}(1)
\end{aligned}
$$

where

$$
\begin{aligned}
& y_{0}(1)=R\left[\Delta y_{0}: \Delta x_{0}\right] \\
& y_{1}(1)=R\left[\Delta y_{1}: \Delta x_{1}\right] \\
& x_{0}{ }^{2}(1)=R\left[\Delta x_{0}{ }^{2}: \Delta x_{0}\right] \\
& x_{1}{ }^{2}(1)=R\left[\Delta x_{1}{ }^{2}: \Delta x_{1}\right]
\end{aligned}
$$

This yield

$$
\Delta y_{0}(1)=c\left\{x_{1}^{2}(1)-x_{0}^{2}(1)\right\}
$$




$$
\text { i.e. } c=y_{0}(2) \text { where } y_{0}(2)=R\left[\Delta y_{0}(1): \Delta x_{0}^{2}(1)\right]
$$

Putting the value of $c$ in any one of the two equations $(2.10-a) \&(2.10-b)$, the value of $b$ can be obtained. The value of $b$ is

$$
b=y_{0}(1)-y_{0}(2) x_{0}{ }^{2}(1)=y_{1}(1)-y_{0}(2) x_{1}{ }^{2}(1)
$$

Finally putting the value of $b \& \mathrm{c}$ in any one of the three equations $(2.9-a),(2.9-b) \&(2.9-c)$, the value of $a$ can be obtained. The value of $a$ thus obtained is

$$
a=y_{0}-b x_{0}-c x_{0}^{2}=y_{1}-b x_{1}-c x_{1}^{2}=y_{2}-b x_{2}-c x_{2}^{2}
$$

with $b \& c$ given by equations (2.13) \& (2.12) respectively.

Case-3 (Representation of four points by a cubic curve):

Let

$$
\left(x_{0}, y_{0}\right),\left(x_{1}, y_{1}\right),\left(x_{2}, y_{2}\right),\left(x_{3}, y_{3}\right)
$$

be four points in $X-Y$ plane.

Let the $3^{\text {rd }}$ degree curve passing through the four points be

$$
y=a+b x+c x^{2}+d x^{2}
$$

where $a, b, c \& d$ are parameters.

In this case,

$$
y_{i}=a+b x_{i}+c x_{i}^{2}+d x_{i}^{3} \quad, \quad(i=0,1,2,3)
$$

This yield

$$
\Delta y_{i}=b \Delta x_{i}+c \Delta x_{i}^{2}+d \Delta x_{i}^{3}
$$

$$
\text { i.e. } y_{i}(1)=b+c x_{i}^{2}(1)+d x_{i}^{3}(1)
$$

for $i=0,1,2$.

where

$$
\begin{aligned}
& y_{i}(1)=R\left[\Delta y_{i}: \Delta x_{i}\right] \\
& x_{i}^{2}(1)=R\left[\Delta x_{i}^{2}: \Delta x_{i}\right] \\
& \& x_{i}^{3}(1)=R\left[\Delta x_{i}^{3}: \Delta x_{i}\right]
\end{aligned}
$$

for $i=0,1,2$.

This yields

$$
\Delta y_{i}(1)=c \Delta x_{i}^{2}(1)+d \Delta x_{i}^{3}(1)
$$




$$
\text { i.e. } y_{i}(2)=c+d \Delta x_{i}^{3}(2)
$$

for $i=0,1$.

where

$$
\begin{gathered}
y_{i}(2)=R\left[\Delta y_{i}(1): \Delta x_{i}{ }^{2}(1)\right] \\
\& x_{i}{ }^{3}(2)=R\left[\Delta x_{i}{ }^{3}(1): \Delta x_{i}{ }^{2}(1)\right]
\end{gathered}
$$

for $i=0,1$.

This further yield

$$
\begin{aligned}
& \quad \Delta y_{0}(2)=d \Delta x_{i}{ }^{3}(2) \\
& \text { i.e. } \quad d=y_{0}(3) \text { where } y_{0}(3)=R\left[\Delta y_{0}(2): \Delta x_{0}{ }^{2}(2)\right]
\end{aligned}
$$

Substituting the value of $d$ in any of the two equations given by (2.19) and then solving it one can obtain the value of $c$.

$$
c=y_{0}(2)-y_{0}(3) \Delta x_{0}{ }^{3}(2)=y_{1}(2)-y_{0}(3) \Delta x_{1}{ }^{3}(2)
$$

Next, substituting the values of $d \& c$ in any of the three equations given by (2.17) and then solving it one can obtain the value of $b$.

$b=y_{0}(1)-c x_{0}{ }^{2}(1)-d x_{0}{ }^{3}(1)=y_{1}(1)-c x_{1}{ }^{2}(1)-d x_{1}{ }^{3}(1)=y_{2}(1)-c x_{2}{ }^{2}(1)-d x_{2}{ }^{3}(1)$

Finally, substituting the values of $d, c \& b$ in any of the four equations given by (2.16) and then solving it one can obtain the value of $a$.

$$
\begin{aligned}
a & =y_{0}-b x_{0}-c x_{0}{ }^{2}-d x_{0}{ }^{3}=y_{1}-b x_{1}-c x_{1}{ }^{2}-d x_{1}{ }^{3}=y_{2}-b x_{2}-c x_{2}{ }^{2}-d x_{2}{ }^{3} \\
& =y_{3}-b x_{3}-c x_{3}{ }^{2}-d x_{3}{ }^{3}
\end{aligned}
$$

Case-4 (Representation of four points by a polynomial curve of degree four):

Let

$$
\left(x_{0}, y_{0}\right),\left(x_{1}, y_{1}\right),\left(x_{2}, y_{2}\right),\left(x_{3}, y_{3}\right),\left(x_{4}, y_{4}\right)
$$

be five points in $X-Y$ plane.

Let the $4^{\text {th }}$ degree curve passing through the five points be

$$
y=a+b x+c x^{2}+d x^{3}+e x^{4}
$$

where $a, b, c, d \& e$ are constants.

In this case,

$$
y_{i}=a+b x_{i}+c x_{i}^{2}+d x_{i}^{3}+e x_{i}^{4},(i=0,1,2,3,4)
$$


This yield

$$
\begin{aligned}
\Delta y_{i} & =b \Delta x_{i}+c \Delta x_{i}{ }^{2}+d \Delta x_{i}{ }^{3}+e \Delta x_{i}{ }^{4} \\
\text { i.e. } \quad y_{i}(1) & =b+c x_{i}{ }^{2}(1)+d x_{i}{ }^{3}(1)+e x_{i}{ }^{4}(1)
\end{aligned}
$$

for $i=0,1,2,3$.

where

$$
\begin{aligned}
y_{i}(1) & =R\left[\Delta y_{i}: \Delta x_{i}\right], \\
x_{i}{ }^{2}(1) & =R\left[\Delta x_{i}^{2}: \Delta x_{i}\right], \\
x_{i}{ }^{3}(1) & =R\left[\Delta x_{i}^{3}: \Delta x_{i}\right] \\
\& x_{i}{ }^{4}(1) & =R\left[\Delta x_{i}^{4}: \Delta x_{i}\right]
\end{aligned}
$$

for $i=0,1,2,3$.

These yields

$$
\Delta y_{i}(1)=c \Delta x_{i}{ }^{2}(1)+d \Delta x_{i}^{3}(1)+e \Delta x_{i}^{4}(1)
$$

$$
\text { i.e. } y_{i}(2)=c+d \Delta x_{i}^{3}(2)+e \Delta x_{i}^{4}(2)
$$

for $i=0,1,2$

where

$$
\begin{aligned}
y_{i}(2) & =R\left[\Delta y_{i}(1): \Delta x_{i}^{2}(1)\right] \\
x_{i}{ }^{3}(2) & =R\left[\Delta x_{i}^{3}(1): \Delta x_{i}^{2}(1)\right] \\
\& \quad x_{i}^{4}(2) & =R\left[\Delta x_{i}^{4}(1): \Delta x_{i}^{2}(1)\right]
\end{aligned}
$$

for $i=0,1,2$.

This further yield

$$
\begin{aligned}
\Delta y_{i}(2) & =d \Delta x_{i}^{3}(2)+e \Delta x_{i}^{4}(2) \\
\text { i.e. } y_{i}(3) & =d+e x_{i}^{4}(3), i=0,1
\end{aligned}
$$

where

$$
\begin{aligned}
& y_{i}(3)=R\left[\Delta y_{i}(2): \Delta x_{i}{ }^{3}(2)\right], i=0,1 \\
& \& \quad x_{i}{ }^{4}(3)=R\left[\Delta x_{i}{ }^{4}(2): \Delta x_{i}{ }^{3}(2)\right], i=0,1
\end{aligned}
$$

This further yield

$$
\begin{gathered}
\Delta y_{0}(3)=e \Delta x_{0}{ }^{4}(3) \\
\text { i.e. } e=R\left[\Delta y_{i}(3): \Delta x_{i}^{4}(3)\right]
\end{gathered}
$$

Substituting the value of $e$ in any of the two equations given by (2.28) and then solving it one can obtain the value of $d$. 
Next, substituting the values of $e \& d$ in any of the three equations given by (2.26) and then solving it one can obtain the value of $c$.

Next, substituting the values of $e, d \& c$ in any of the four equations given by (2.24) and then solving it one can obtain the value of $b$.

Finally, substituting the values of $e, d, c \& b$ in any of the five equations given by (2.23) and then solving it one can obtain the value of $a$.

Case-5 (General Case - Representation of $(n+1)$ points by a polynomial curve of degree $n$ ):

Let

$$
\left(x_{0}, y_{0}\right),\left(x_{1}, y_{1}\right),\left(x_{2}, y_{2}\right), \ldots \ldots \ldots \ldots . .,\left(x_{n-1}, y_{n-1}\right),\left(x_{n}, y_{n}\right)
$$

be n points in $X-Y$ plane.

The objective is to represent these $\mathrm{n}$ points by a polynomial of the form

$$
y=f(x)=a_{0}+a_{1} x+a_{2} x^{2}+a_{2} x^{3}+\ldots \ldots \ldots+a_{n} x^{n}, a_{n} \neq 0
$$

where $a_{0}, a_{1}, a_{2}, a_{3} \ldots \ldots \ldots \ldots \ldots, a_{n}$ are constants.

Since the $(n+1)$ points pass through the curve

therefore,

$y_{i}=a_{0}+a_{1} x_{i}+a_{2} x_{i}^{2}+a_{3} x_{i}^{3}+$ $+a_{n} x_{i}^{n}, a_{n} \neq 0$

$(i=0,1,2,3$,

$, n)$.

This yield

$$
\begin{array}{ll} 
& \Delta y_{i}=a_{1} \Delta x_{i}+a_{2} \Delta x_{i}^{2}++a_{3} \Delta x_{i}^{3}+\ldots \ldots \ldots .+a_{n} \Delta x_{i}^{n} \\
\text { i.e. } & y_{i}(1)=a_{1}+a_{2} x_{i}^{2}(1)++a_{3} x_{i}^{3}(1)+\ldots \ldots .+a_{n} x_{i}^{n}(1)
\end{array}
$$

where

$$
\begin{aligned}
& y_{i}(1)=R\left[\Delta y_{i}: \Delta x_{i}\right] \text {, } \\
& x_{i}^{2}(1)=R\left[\Delta x_{i}^{2}: \Delta x_{i}\right] \text {, } \\
& x_{i}^{3}(1)=R\left[\Delta x_{i}^{3}: \Delta x_{i}\right] \text {, } \\
& x_{i}{ }^{n}(1)=R\left[\Delta x_{i}{ }^{n}: \Delta x_{i}\right] \text {, }
\end{aligned}
$$

$(i=0,1,2,3$, $, n-1)$

This yields 


$$
\begin{aligned}
& \Delta y_{i}(1)=a_{2} x_{i}{ }^{2}(1)+a_{3} \Delta x_{i}{ }^{3}(1)+\ldots \ldots \ldots \ldots \ldots+a_{n} \Delta x_{i}{ }^{n}(1) \\
& \text { i.e. } y_{i}(2)=a_{2}+a_{3} x_{i}{ }^{3}(2)+\ldots \ldots \ldots \ldots \ldots+a_{n} x_{i}{ }^{n}(2) \\
&
\end{aligned}
$$

where

$$
\begin{aligned}
& y_{i}(2)=R\left[\Delta y_{i}(1): \Delta x_{i}^{2}(1)\right] \text {, } \\
& x_{i}^{3}(2)=R\left[\Delta x_{i}^{3}(1): \Delta x_{i}^{2}(1)\right] \text {, } \\
& x_{i}{ }^{n}(2)=R\left[\Delta x_{i}^{n}(1): \Delta x_{i}{ }^{2}(1)\right] \text {, }
\end{aligned}
$$

$(i=0,1,2,3$,

$$
n-2) \text {. }
$$

This yield

$$
\Delta y_{i}(2)=a_{2}+a_{3} \Delta x_{i}{ }^{3}(2)+\ldots \ldots \ldots \ldots \ldots . .+a_{n} \Delta x_{i}{ }^{n}(2)
$$

i.e. $y_{i}(3)=a_{3}+a_{4} x_{i}^{4}(3)+\ldots \ldots . .+a_{n-1} x_{i}{ }^{n-1}(3)+a_{n} x_{i}{ }^{n}(3)$

where

$$
\begin{aligned}
& y_{i}(3)=R\left[\Delta y_{i}(2): \Delta x_{i}^{3}(2)\right] \text {, } \\
& x_{i}^{4}(3)=R\left[\Delta x_{i}^{4}(2): \Delta x_{i}^{3}(2)\right] \text {, } \\
& x_{i}^{n-1}(3)=R\left[\Delta x_{i}^{n-1}(2): \Delta x_{i}^{3}(2)\right] \text {, } \\
& x_{i}^{n}(3)=R\left[\Delta x_{i}^{n}(2): \Delta x_{i}^{3}(2)\right] \text {, }
\end{aligned}
$$

$(i=0,1,2,3$,

$$
, n-3) \text {. }
$$

At the $k^{\text {th }}$ step, one can obtain that

$$
\begin{array}{ll} 
& \Delta y_{i}(k-1)=a_{k} \Delta x_{i}{ }^{k}(k-1)+a_{k+1} \Delta x_{i}{ }^{k+1}(k-1)+\ldots \ldots \ldots \ldots \ldots+a_{n} \Delta x_{i}{ }^{n}(k-1) \\
\text { i.e. } & y_{i}(k)=a_{k}+a_{k+1} \Delta x_{i}{ }^{k+1}(k)+\ldots \ldots \ldots \ldots \ldots+a_{n} \Delta x_{i}{ }^{n}(k)
\end{array}
$$
$+a_{n} \Delta x_{i}^{n}(k)$

where

$$
\begin{aligned}
& y_{i}(k)=R\left[\Delta y_{i}(k-1): \Delta x_{i}^{k}(k-1)\right], \\
& x_{i}{ }^{k+1}(k)=R\left[\Delta x_{i}{ }^{k+1}(k-1): \Delta x_{i}{ }^{k}(k-1)\right] \text {, } \\
& x_{i}{ }^{k+2}(k)=R\left[\Delta x_{i}{ }^{k+2}(k-1): \Delta x_{i}{ }^{k}(k-1)\right] \text {, } \\
& x_{i}^{n-1}(k)=R\left[\Delta x_{i}^{n-1}(k-1): \Delta x_{i}{ }^{k}(k-1)\right], \\
& x_{i}^{n}(k)=R\left[\Delta x_{i}^{n}(k-1): \Delta x_{i}^{k}(k-1)\right] \text {, } \\
& , n-k) \text {. }
\end{aligned}
$$$$
(i=0,1,2,3 \text {, }
$$ 
Continuing the process, one can arrive at the $(n-1)^{\text {th }}$ step that

$$
\begin{aligned}
\Delta y_{i}(n-2) & =a_{n-1} \Delta x_{i}^{n-1}(n-2)+a_{n} \Delta x_{i}^{n}(n-2) \\
\text { i.e. } \quad y_{i}(n-1) & =a_{n-1}+a_{n} x_{i}^{n}(n-1)
\end{aligned}
$$

where

$$
y_{i}(n-1)=R\left[\Delta y_{i}(n-2): \Delta x_{i}^{n-1}(n-2)\right] \quad, \quad(i=0,1)
$$

At the $\mathrm{n}^{\text {th }}$ step, one obtain that

$$
\Delta y_{0}(n-1)=a_{n} \Delta x_{0}^{n}(n-1)
$$

i.e. $a_{n}=y_{0}(n)$ where $y_{0}(n)=R\left[\Delta y_{0}(n-1): \Delta x_{0}{ }^{n}(n-1)\right]$

Substituting the value of $a_{n}$ in any of the two equations given by (2.37) and then solving it one can obtain the value of $a_{n-1}$.

Next, substituting the values of $a_{n} \& a_{n-1}$ in any of the three equations, containing $a_{n}, a_{n-1}, a_{n-2}$, obtained in the just earlier steps one can obtain the value of $a_{n-2}$.

Similarly in the next step, substituting the values of $a_{n}, a_{n-1}, a_{n-2}$, in any of the four equations, containing $a_{n}, a_{n-1}, a_{n-2} \& a_{n-3}$ obtained in the just earlier steps one can obtain the value of $a_{n-3}$. Continuing the process one can obtain the values of the parameters in the order

$$
a_{n}, a_{n-1}, a_{n-2}, \ldots \ldots \ldots \ldots . . ., a_{3}, a_{2}, a_{1}, a_{0}
$$

\section{Numerical Example:}

Example 3.1: The following table shows the data on total population of India at four different years of interval ten years:

\begin{tabular}{|c|c|c|c|c|}
\hline Year & 1971 & 1981 & 1991 & 2001 \\
\hline Total Population & 548159652 & 683329097 & 846302688 & 1027015247 \\
\hline
\end{tabular}

Taking 1971 as origin and changing scale by 1/10, one can obtain the following table for independent variable $x$ (representing time) and $\mathrm{f}(\mathrm{x})$ (representing total population of India):

\begin{tabular}{|c|c|c|c|c|}
\hline Year & 1971 & 1981 & 1991 & 2001 \\
\hline$x_{i}$ & 0 & 1 & 2 & 3 \\
\hline$y_{i}=f\left(x_{i}\right)$ & 548159652 & 683329097 & 846302688 & 1027015247 \\
\hline
\end{tabular}


It is wanted to represent these data by a cubic curve defined by equation (2.15).

In this case,

$$
\begin{aligned}
n & =3, x_{0}=0, x_{1}=1, x_{2}=2 \& x_{3}=3, \\
y_{0} & =f\left(x_{0}\right)=548159652, y_{1}=f\left(x_{1}\right)=683329097, \\
y_{2} & =f\left(x_{2}\right)=846302688 \quad \& \quad y_{3}=f\left(x_{3}\right)=1027015247 .
\end{aligned}
$$

Applying the equation (2.21), it is found that

$$
d=-1677529.67
$$

Substituting this value of $d$ in any one equation of (2.19), it is found that

$$
c=18934662
$$

Next substituting these two values of $d \& c$ in any one equation of (2.17), it is found that

$$
b=117912312.7
$$

Finally substituting these three values of $d, c \& b$ in any one equation of (2.16), it is found that

$$
a=548159652
$$

Hence, the equation of the cubic curve that can represent the given numerical data is

$$
y=f(x)=-1677529.67 x^{3}+18934662 x^{2}+117912312.7 x+548159652
$$

This curve can yield the values of the function $y=f(x)$ corresponding to the respective observed values of $x$ as follows:

$$
\begin{aligned}
f(0) & =548159652, \\
f(1) & =683329097, \\
f(2) & =846302687.6 \\
\& f(3) & =1027015246.1
\end{aligned}
$$

It is observed that these values yielded by the equation of the polynomial curve are identical with the respective observed values.

Example 3.2: Now let us consider the data on total population of India at five successive years of interval ten years:

\begin{tabular}{|c|c|c|c|c|c|}
\hline Year & 1971 & 1981 & 1991 & 2001 & 2011 \\
\hline Total Population & 548159652 & 683329097 & 846302688 & 1027015247 & 1210193422 \\
\hline
\end{tabular}


Taking 1971 as origin and changing scale by 1/10, one can obtain the following table for independent variable $\mathrm{x}$ (representing time) and $y=f(x)$ (representing total population of India):

\begin{tabular}{|c|c|c|c|c|c|}
\hline Year & 1971 & 1981 & 1991 & 2001 & 2011 \\
\hline$x_{i}$ & 0 & 1 & 2 & 3 & 4 \\
\hline$y_{i}=f\left(x_{i}\right)$ & 548159652 & 683329097 & 846302688 & 1027015247 & 1210193422 \\
\hline
\end{tabular}

It is wanted to represent these data by a polynomial curve of degree four defined by equation (2.22).

In this case,

$$
\begin{gathered}
n=4, x_{0}=0, x_{1}=1, x_{2}=2, x_{3}=3, x_{4}=4, \\
y_{0}=f\left(x_{0}\right)=548159652, y_{1}=f\left(x_{1}\right)=683329097, y_{2}=f\left(x_{2}\right)=846302688, \\
y_{3}=f\left(x_{3}\right)=1027015247 \& y_{4}=f\left(x_{4}\right)=1210193422 .
\end{gathered}
$$

Applying the equation (2.30), it is found that

$$
e=-217007.25
$$

Substituting this value of $e$ in any one equation of (2.28), it is found that

$$
d=-375486.2
$$

Next substituting these two values of $d \& e$ in any one equation of (2.26), it is found that

$$
c=16547582.37
$$

Next substituting these three values of $e, d \& c$ in any one equation of (2.24), it is found that

$$
b=119214356
$$

Next substituting these three values of $e, d, c \& b$ in any one equation of (2.23), it is found that

$$
a=548159652
$$

Hence, the equation of the polynomial curve of degree four that can represent the given numerical data is

$$
y=f(x)=-217007.25 x^{4}-375486.2 x^{3}+16547582.3 x^{2}+119214356 x+548159652
$$

The values of the function $y=f(x)$ corresponding to the obse4rved values of $x$ yielded by this polynomial curve are as follows:

$$
\begin{gathered}
f(0)=548159652, \\
f(1)=683329097 \\
f(2)=846302687.6
\end{gathered}
$$




$$
\begin{gathered}
f(3)=1027015246.1 \\
\& f(4)=1210193432 .
\end{gathered}
$$

These values are found to be identical with the corresponding observed values of the function $y=f(x)$.

\section{Conclusion:}

The method developed here, based on two numerical operations namely finite difference operation and ratio operation, for representing a given set of numerical data on a pair of variables by a suitable polynomial is a systematic and disciplined one. It is simpler than the other methods, developed till now, for representing a given set of numerical data on a pair of variables by a polynomial.

In selecting the degree of the polynomial for representing a given set of data on a pair of variables, it is to be noted that the degree of the polynomial is one less than the number of pairs of observations. The polynomial that represents the given set of numerical data can be used for interpolation at any position of the independent variable lying within its two extreme values.

The method, developed here, can also be suitably applied in inverse interpolation also. Moreover, the method can suitably be applied in extrapolation corresponding to a value of the independent variable which lies outside its two extremes of the available values.

\section{Reference:}

Bathe K. J. \& Wilson E. L., "Numerical Methods in Finite Element Analysis", Prentice-Hall, Englewood Cliffs, NJ, 1976.

Chakrabarty D., "Representation of Numerical Data on a Pair of Variables by a Polynomial Curve Expressed in the Simplest Form", International Journal of Electronics and Applied Research (ISSN : 2395 - 0064), 3(1), 26 - 39, $2016 a$.

Chakrabarty D., "Interpolation: One Method of Representation of Numerical Data on a Pair of Variables by a Polynomial Curve Expressed In the Simplest Form", Journal of Environmental Science, Computer Science and Engineering \& Technology, Section C, (E-ISSN : 2278 - 179 X), 5(3), 405 - 418, $2016 b$.

Chakrabarty D., "Difference and Ratio Operators: Representation of Numerical Data on a Pair of Variables by a Polynomial Curve", Journal of Environmental Science, Computer Science and Engineering \& Technology, Section C, (E-ISSN : 2278 - 179 X), 5(4), $549-560,2016 c$. 
Chakrabarty D., "Recent Developments on Representation of Numerical Data by a Polynomial Curve", International Journal of Electronics and Applied Research (ISSN : 2395 - 0064), 3(2), 125 - 158, $2016 \mathrm{~d}$.

Chakrabarty D., "Backward Difference and Ratio Operations: Representation of Numerical Data on a Pair of Variables by a Polynomial Curve", Journal of Environmental Science, Computer Science and Engineering \& Technology, Section C, (E-ISSN : 2278 - 179 X), 6(1), 027 -039, 2016 - 17.

Chakrabarty D., "Some Forms of Interpolation Formula Based on Divided Difference", Journal of Environmental Science, Computer Science and Engineering \& Technology, Section C, (E-ISSN : 2278 179 X), 6(2), $199-211,2017 a$.

Chakrabarty D., "Representation of Numerical Data by Some Special Mathematical Curves", International Journal of Electronics and Applied Research (ISSN : 2395 - 0064), 4(1), 52 - 74, $2017 b$.

Das B. \& Chakrabarty D., "Lagrange's Interpolation Formula: Representation of Numerical Data by a Polynomial Curve", International Journal of Mathematics Trends and Technology (IJMTT) (ISSN: 2231 - 5373), 34(2), $64-72,2016 a$.

Das B. \& Chakrabarty D., "Newton's Divided Difference Interpolation Formula: Representation of Numerical Data by a Polynomial Curve", International Journal of Mathematics Trends and Technology (IJMTT) (ISSN: $2231-5373), 35(3), 197-203,2016 b$.

Das B. \& Chakrabarty D., 'Newton's Forward Interpolation: Representation of Numerical Data by a Polynomial Curve", International Journal of Statistics and Applied Mathematics (IJMTT) (ISSN: 2456 1452), 1(2), $36-41,2016 c$.

Das B. \& Chakrabarty D., "Newton's Backward Interpolation: Representation of Numerical Data by a Polynomial Curve", International Journal of Applied Research (IJAR) (ISSN Print: 2394-7500, ISSN Online: 2394-5869), 2(10), 513 - 517, 2016d.

Das B. \& Chakrabarty D., "Matrix Inversion: Representation of Numerical Data by a Polynomial Curve", Aryabhatta Journal of Mathematics \& Informatics \{ISSN (Print) : 0975-7139, ISSN (Online) : 23949309\}, 8(2), $267-276,2016 e$.

Das B. \& Chakrabarty D., "Inversion of Matrix by Elementary Transformation: Representation of Numerical Data by a Polynomial Curve", Journal of Mathematics and Systems Sciences (ISSN : 0975 5454), 12(1-2), $27-32,2016 f$.

Das B. \& Chakrabarty D., "Inversion of Matrix by Elementary Column Transformation: Representation of Numerical Data by a Polynomial Curve", International Journal of Mathematics Trends and Technology (IJMTT) (ISSN: 2231 - 5373), 42(1), 45 - 49, $2017 a$.

Das B. \& Chakrabarty D., "Backward Divided Difference: Representation of Numerical Data by a Polynomial Curve", International Journal of Statistics and Applied Mathematics (ISSN: 2456 - 1452), 2(2), $1-6,2017 b$. 
Das B. \& Chakrabarty D., "Representation of Numerical Data by Exponential Curve", Aryabhatta Journal of Mathematics \& Informatics \{ISSN (Print) : 0975-7139, ISSN (Online) : 2394-9309\}, 9(1), 157 $-162,2017 c$.

Das B. \& Chakrabarty D., "Representation of Numerical Data by Modified Exponential Curve", Journal of Mathematics and Systems Sciences (ISSN : 0975 - 5454), 13(1-2), 1 - 6, 2017d.

Das B., Chakrabarty D. \& Kashyap M. P., "Representation of Numerical Data by Makeham's Curve and Third Degree Polynomial Curve: A Comparative Study", International Journal of Advanced Research in Science, Engineering and Technology, (ISSN : 2350 - 0328), 4(11), 4845 - 4853, 2017.

Das B., Chakrabarty D. \& Kashyap M. P., "Population in India: Suitability of Logistic, Modified Exponential and Second Degree Parabolic Curves", Journal of Environmental Science, Computer Science and Engineering \& Technology, Section C, (E-ISSN : 2278 - 179 X), 7(2), 107 - 116, 2018.

Das B. \& Chakrabarty D., "Numerical Data on a Pair of Variables: Representation by Logistic Curve", International Journal of Advanced Research in Science, Engineering and Technology, (ISSN : 2350 0328), 5(6), $6113-6119,2018 a$.

Das B. \& Chakrabarty D., "Numerical Data on a Pair of Variables: Representation by Makeham's Curve", Aryabhatta Journal of Mathematics \& Informatics \{ISSN (Print) : 0975-7139, ISSN (Online) : 2394-9309\}, 10(2), $277-286,2018 b$.

Echols W. H., "On Some Forms of Lagrange's Interpolation Formula”, "The Annals of Mathematics", Jstor, 8(1/6), 22 - 24, 1893.

Erdos P. \& Turan P., "On Interpolation II: On the Distribution of the Fundamental Points of Lagrange and Hermite Interpolation”, “The Annals of Mathematics, 2nd Ser", Jstor, 39(4), 703 - 724, 1938.

Hummel P. M., "A Note on Interpolation (in Mathematical Notes)", "American Mathematical Monthly", Jstor, 54(4), 218 - 219, 1947.

Mills T. M., "An introduction to analysis by Lagrange interpolation”, "Austral. Math. Soc. Gaz.”, 4(1), MathSciNet, 10 - 18, 1977.

Quadling D. A., "Lagrange's Interpolation Formula", “The Mathematical Gazette”, L(374), 372 - 375, 1966.

Revers \& Michael B., "On Lagrange interpolation with equally spaced nodes", "Austral. Math. Soc MathSciNet.", 62(3), 357-368, 2000.

Traub J. F., "On Lagrange-Hermite Interpolation", “Journal of the Society for Industrial and Applied Mathematics", Jstor , 12(4), 886 - 891, 1964. 
Wisniewski J. K., "Note on Interpolation (in Notes)", Journal of the American Statistical Association, 25(170), $203-205,1930$.

Whittaker E. T. \& Robinson G., "Lagrange's Formula of Interpolation", "The Calculus of Observations: A Treatise on Numerical Mathematics, 4th ed.”, §17, New York: Dover, 28 - 30, 1967. 